\title{
Automated high-throughput sex-typing assay
}

\author{
Lizhi Yu, Fernando D. Martinez, and Walter T. Klimecki \\ Arizona Respiratory Center, Tucson, Arizona, USA
}

BioTechniques 37:662-664 (October 2004)

Determining the sex of the donor of a human DNA sample in a rapid and accurate manner is of importance in several settings, including basic research, clinical practice, and forensics $(1,2)$. Our group routinely performs high-throughput genotyping within large epidemiological studies. It is not uncommon to receive thousands of DNA samples, with the tube identification as the only accompanying information. These samples will then be distributed through a chain of multi-well plates, ultimately ending in 384-well PCR plates in which there is no individual identification for a given sample, only a plate identifier. In such cases, a basic test of incoming sample identification integrity and of sample processing/tracking integrity is to sex type both the original DNA sample tubes as well as the final 384-well reaction plates. These data are compared to the sex of the study participant, which is recorded in the database storing the epidemiological information, providing basic surveillance for significant sample tracking and identification problems.

Recently, Gold et al. (3) published a multiplex PCR-based assay designed to produce a 475 -bp fragment from the $A B C D 1$ gene (X-chromosome) and a 231-bp fragment from the $S R Y$ gene (Y-chromosome). As the authors point out, this assay design has significant advantages, including the lack of nested primers and genomic targeting to unique, reliable sequences. In this report, we describe a significant refinement of this assay, in which these genetic regions are targeted in an endpoint-based $5^{\prime}$-exonuclease $\left(\right.$ TaqMan $\left.^{\circledR}\right)$ assay. This assay takes advantage of the 5 -exonuclease activity of Taq DNA polymerase (Applied Biosystems, Foster City, CA, USA) to cleave the reporter fluorescent molecule from the $5^{\prime}$ end of a probe that is hybridized $3^{\prime}$ to an extending PCR primer on a template strand of DNA. A key strength of this assay is that it is a homogenous reac- tion, requiring only one reaction setup and no subsequent reaction processing. Notably, this assay does not rely on a cumbersome electrophoretic analysis. This improvement, coupled with a 384-well reaction plate format, allows the assay to be performed in a highthroughput automated setting.

Oligonucleotides were designed according to default parameters using Primer Express ${ }^{\circledR}$ software (Applied Biosystems). PCR primer and probe sequences are described in Table 1 and were synthesized by Integrated DNA Technologies (Coralville, IA, USA). Both probes contained the Black Hole Quencher-1 ${ }^{\mathrm{TM}}$ (BHQ-1) fluorescence quencher at their $3^{\prime}$ ends. TaqMan reactions were performed in $5 \mu \mathrm{L}$ volumes, consisting of the following final concentrations: $900 \mathrm{nM}$ of each PCR primer (of 4 primers), $200 \mathrm{nM}$ of each probe (of 2 probes), $1 \times$ TaqMan Universal Master Mix (Applied Biosystems), and $5 \mathrm{ng} / \mu \mathrm{L}$ sample DNA. Reactions were thermal cycled in 384-well plates using a GeneAmp ${ }^{\circledR}$ PCR System 9700 (Applied Biosystems) at $95^{\circ} \mathrm{C}$ for $10 \mathrm{~min}$, and then 40 cycles of $95^{\circ} \mathrm{C}$ for $15 \mathrm{~s}$ and $58^{\circ} \mathrm{C}$ for $1 \mathrm{~min}$. Following thermal cycling, the plates were analyzed on an ABI PRISM ${ }^{\circledR}$ 7900HT Sequence Detection System (Applied Biosystems). Data were analyzed using SDS 2.0 software (Applied Biosystems). Technicians were blinded as to the actual sex of the samples prior to assigning data clusters to four categories, male, female, no template control, and outlier. The no template control group was identified as the cluster closest to the origin of the $\mathrm{x}-(A B C D 1 / \mathrm{FAM}$ fluorescence) and y- (SRY/TET fluorescence) axes. The male cluster was identified as having high $\mathrm{x}$-axis and $\mathrm{y}$-axis fluorescence. The female cluster was identified as having high $\mathrm{x}$-axis fluorescence and low y-axis fluorescence. Outliers were defined as data points that could not be assigned to one of the three defined clusters.
As a means of evaluating the performance of this assay, a total of 140 DNA samples were tested repeatedly in 3 independent experiments that were assembled on different days by different technicians. The entire reaction setup process was done with a robotic liquid transferring system (Multiprobe ${ }^{\circledR}$ II HT EX; Perkin Elmer, Boston, MA, USA), with the exception of the initial preparation of the reagent, which was assembled manually. To verify the accuracy of the TaqMan assay, the data were compared with the sample sex information from the source of the DNA samples (Corielle Institute for Medical Research, Camden, NJ, USA).

To establish that the primer and probe binding sites that were targeted in this assay are not polymorphic, we resequenced this genomic region in 24 DNA samples of African ancestry and 116 DNA samples of European ancestry that were studied in this report. Genomic sequences for $A B C D 1$ and $S R Y$ were accessed from the University of California, Santa Cruz (UCSC) Genome Browser (http://www.genome.ucsc.edu), July 2003 freeze. PCR products were designed to position the TaqMan primer and probe binding sites in the middle of the products. PCRs contained $20 \mathrm{ng}$ genomic DNA, 1 pmol of each primer, 0.2 U Taq DNA polymerase (Platinum ${ }^{\circledR}$ Taq; Invitrogen, Carlsbad, CA, USA), and $0.1 \mu \mathrm{M}$ dNTPs in a total volume of $10 \mu \mathrm{L}$. PCR products were prepared for cycle sequencing by diluting them with water, using a dilution factor of $1: 3$. Cycle sequencing reactions were assembled using $0.4 \mu \mathrm{L}$ cycle sequencing premixture (ABI PRISM BigDye ${ }^{\mathrm{TM}}$ Terminators v3.0 Cycle Sequencing Kit; Applied Biosystems), 1 pmol sequencing primer, $1.8 \mu \mathrm{L} 5 \times$ sequencing buffer, and $2 \mu \mathrm{L}$ PCR product in a final volume of $10 \mu \mathrm{L}$. Cycle sequencing reactions were purified using CleanSEQ ${ }^{\circledR}$ DNAaffinity magnetic beads (Agencourt Biosciences, Beverly, MA, USA). Purified sequencing reactions were electrophoretically analyzed using a DNA Analyzer 3730 (Applied Biosystems). Sequence chromatograms were processed for base calling and assembly using the Phred, Phrap, and Consed suite of software programs (http://phrap.org). Chromatograms at potential polymorphic sites, initially identified using the 
Table 1. Oligonucleotide Composition for Sex-Determination Assay

\begin{tabular}{|ll|}
\hline SRY & Sequence \\
\hline PCR Forward & 5'-TTGTAGCCAATGTTACCCGATT-3' \\
PCR Reverse & 5'-AGGGATGACTGTACGAAAGCC-3' \\
Probe (TET-Labeled) & 5'-TGGCTGTAGCGGTCCCGTTGCT-3' \\
ABCD1 & \\
\hline PCR Forward & 5'-AGCTGGCATGAACCGGGTAT-3' \\
PCR Reverse & 5'-ACAGGAAGGTGCGGCTCA-3' \\
Probe (FAM-Labeled) & 5'-TGTGGCTCCTGCGGCTGCTGTT-3' \\
\hline
\end{tabular}

Polyphred software program, were visually inspected by trained analysts to distinguish true polymorphic sites from false positives identified by the software program.

In order to quantitate the $S R Y$ and $A B C D 1$ signals from the two outlier samples that we observed within the set of 140 DNA samples in the sex-typing assay, 10 DNA samples (4 males and 4 females according to the vender's sex assignment, together with the 2 outliers found in sex-typing assay) were assayed by real-time PCR assays in nonmultiplexed reactions, using $A B C D 1$ and $S R Y$ probes. Real-time PCRs were assembled in a $20-\mu \mathrm{L}$ volume using 10 ng DNA, $400 \mathrm{nM}$ of each primer (of 2 primers), $80 \mathrm{nM} A B C D 1$ or $S R Y$ probe, and $1 \times$ TaqMan Universal Master Mix. Each sample was analyzed in triplicate reactions. Thermal cycling and fluorescence analysis were performed on the ABI PRISM 7900HT Sequence Detection
System at $95^{\circ} \mathrm{C}$ for $10 \mathrm{~min}$, and then 40 cycles of $95^{\circ} \mathrm{C}$ for $15 \mathrm{~s}$, and $58^{\circ} \mathrm{C}$ for 1 min. Data were analyzed using SDS 2.0 software. The concentration of DNA used in this real-time PCR analysis was determined by first quantitating the DNA in the original sample by a separate real-time PCR designed to assay the concentration of a single-copy gene (human interleukin-10) in an absolute quantification assay, and then diluting the sample according to the real-time PCR-determined concentration.

Cumulative results from three sextyping experiments are shown in Figure 1 . Over the three experiments, the assay produced a sex assignment call rate of $98.6 \%$. Two samples, designated samples $\mathrm{M}$ and $\mathrm{N}$, repeatedly could not be assigned to any clusters. The 138 samples that were assigned to clusters were completely concordant with the sample sex that was provided by the vendor. Fluorescence ratio val-

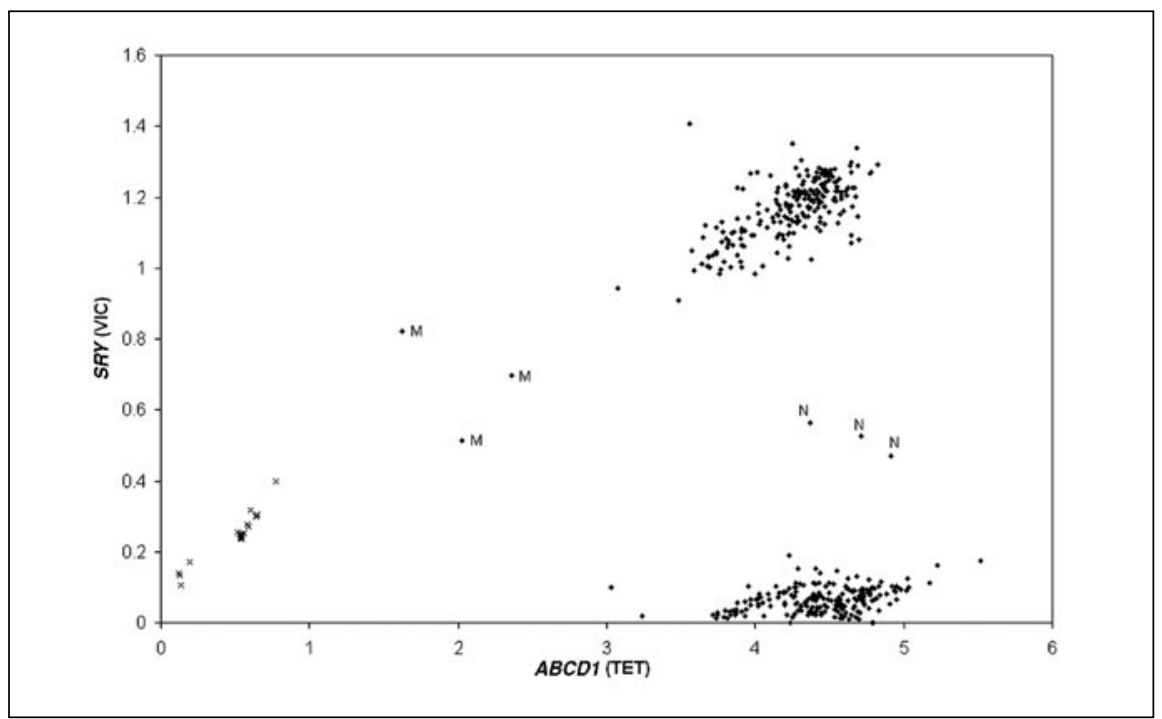

Figure 1. Plot of fluorescence (ratio, normalized to passive standard) for $A B C D 1$ on the $\mathrm{x}$-axis and $S R Y$ on the y-axis. Data of 3 independent experiments on 7 blank (no DNA) samples (shown as X) and 140 DNA samples (shown as ) are overlaid (21 blank and 420 DNA samples total). The data points of subject $\mathrm{M}$ and subject $\mathrm{N}$ are labeled. 
ues (probe reporter/passive standard) for the Y-chromosome marker SRY were the mean $( \pm \mathrm{SD})$ of $1.204 \pm 0.055$ and $0.069 \pm 0.035$ for male and female clusters, respectively. For the male cluster, $A B C D 1$ fluorescence was 4.432 \pm 0.220 , whereas for the female cluster fluorescence was $4.719 \pm 0.248$. This pilot study with 140 DNA samples suggested that the assay could be readily performed with automatic procedures in larger scale without losing the accuracy and reliability. We validated this with a scale-up test sample group of 1000 DNA samples. With the automatic procedures mentioned above, the whole process was done in $2 \mathrm{~h}$, with similar accuracy and call rate as the 140-sample group (data not shown).

We analyzed the primer and probe binding sites by resequencing to establish that these sites are nonpolymorphic in human DNA, which is a requirement for consistent performance of the assay. In total, 24 African ancestry samples and 116 European ancestry samples were analyzed. No polymorphic sites within any primer or probe binding region were detected in any DNA samples. While the sensitivity of polymorphism detection in this sample is fairly definitive for European samples, with a detection frequency of $1.4 \%$ for the $\mathrm{X}$ chromosome and $4.2 \%$ for the Y-chromosome (at a $95 \%$ confidence interval), the data for African ancestry samples should be considered preliminary and be validated in a larger sample set.

Based on the consistency of the fluorescence values for the two outlier samples (Figure 1, designated $\mathrm{M}$ and $\mathrm{N}$ ), we were interested in determining whether the cause of the anomalous fluorescence related to sample preparation as opposed to potential genetic anomalies in the samples themselves. Real-time PCR showed that outlier $\mathrm{M}$, which was recorded as a male by the vender, has a cycle threshold $\left(\mathrm{C}_{\mathrm{t}}\right)$ value of $34.77 \pm 0.14$ for $A B C D 1$ and $\mathrm{a}_{\mathrm{t}}$ value of $27.56 \pm 0.11$ for $S R Y$. Both are consistent with the other 4 male samples evaluated by realtime PCR $(A B C D 1,34.14 \pm 0.60 ; S R Y$, $27.05 \pm 0.15)$, suggesting that outlier $M$ has the normal copy number of $\mathrm{X}$ - and Y-chromosomes. The fact that the fluorescence values for sample $M$ (Figure 1) align to a vector with its origin at the no template control cluster and extend- ing into the large cluster of male samples suggests that this sample may have been over-diluted in the sample preparation process. This possibility was further supported when we manually prepared a new dilution of DNA from this sample from the original stock tube, and it clustered within the male sample cluster (data not shown). This was not true for sample N, which retained its outlier position when re-assayed from a fresh dilution from the stock tube. Outlier N, which was recorded as female by the vender, had a $\mathrm{C}_{\mathrm{t}}$ value of $33.08 \pm 0.25$ for $A B C D 1$, which is consistent with mean $A B C D 1 \mathrm{C}_{\mathrm{t}}$ value of $32.51 \pm 1.17$ for the other female samples. Of interest, sample $\mathrm{N}$ also showed a $\mathrm{C}_{\mathrm{t}}$ value of $30.96 \pm 0.15$ for $S R Y$. For the four control female samples, a $\mathrm{C}_{\mathrm{t}}$ value for $S R Y$ could not be calculated because of the lack of production of probe fluorescence above background levels. Thus, sample $\mathrm{N}$ appeared to contain genuine, measureable amounts of Y-chromosome DNA, although at lower levels than the four male control samples $\left(C_{t}=30.96 \pm\right.$ 0.15 for $\mathrm{N}$ vs. $27.05 \pm 0.15$ for controls). There are several potential explanations for the results from sample $\mathrm{N}$, ranging from true cytogenetic anomalies involving the $\mathrm{X}$ - and Y-chromosomes to cell line or DNA cross-contamination at the vendor, to DNA cross-contamination in our sample processing. We think that the latter scenario is unlikely, given the scale of volume-to-volume contamination that would have had to occur to produce the concentrations of $A B C D 1$ and $S R Y$ that we measured by real-time PCR. Notwithstanding this, these results validate the accuracy and reproducibility of the assay in the face of sample over-dilution and anomalous sample composition. In a real-world setting, samples $\mathrm{M}$ and $\mathrm{N}$ would likely have been retested from fresh samples. Sample M would have fallen back into a definable cluster, and sample $\mathrm{N}$ would have repeated its anomalous results, prompting follow-up testing that could include cytogenetic analysis.

The TaqMan-based assay for Xand Y-chromosome markers, initially described by Gold et al. (3) and subsequently refined in this report, has several strengths, including low falsepositive rates, high call rates, high accuracy, and high repeatability. It also shows quantitative strength for $\mathrm{X}$ - and Y-chromosome copy numbers, which was demonstrated by outlier N. In addition, the assay scales very robustly; we have used it to test populations as large as 1000. A potential limitation of the assay is the subjective nature of defining the cluster boundaries, although this has not proven to be problematic in practice. Because the currently available sequence detection system software requires three genotype clusters for automatic cluster definition, it cannot be used in the automatic mode in this assay, which generates two clusters. We are currently exploring the use of K-means-based automatic cluster definition in this assay.

\section{ACKNOWLEDGMENTS}

The authors acknowledge Yelitza Rodriguez, Kelley J. Kain, and Mischa Guild for technical assistance. This work was funded by the National Heart, Lung, and Blood Institute (NHLBI) grant no. P01HL67672 (to F.D.M. and W.T.K.).

\section{COMPETING INTERESTS STATEMENT}

The authors declare no competing interests.

\section{REFERENCES}

1.Caenazzo, L., E. Ponzano, N.A. Greggio, and P. Cortivo. 1997. Prenatal sexing and sex determination in infants with ambiguous genitalia by polymerase chain reaction. Genet. Test. 1:289-291.

2.Staessen, C., E. Van Assche, H. Joris, M. Bonduelle, M. Vandervorst, I. Liebaers, and A. Van Steirteghem. 1999. Clinical experience of sex determination by fluorescent insitu hybridization for preimplantation genetic diagnosis. Mol. Hum. Reprod. 5:382-389.

3.Gold, B., J. Bergeron, M. LachtermacherTriunfol, and M. Dean. 2001. Human duplex sex determination PCR. BioTechniques 31:28-35.

Received 31 March 2004; accepted 7 June 2004.

Address correspondence to Walt Klimecki, Arizona Respiratory Center, P.O. Box 245030, Tucson, Arizona 85641, USA. e-mail:walt@resp-sci.arizona.edu 